Bulletin of the Section of Logic

Volume 47/3 (2018), pp. 171-185

http://dx.doi.org/10.18778/0138-0680.47.3.03

Berhanu Assaye, Mihret Alemneh and Gerima Tefera

\title{
B-ALMOST DISTRIBUTIVE FUZZY LATTICE
}

\begin{abstract}
The paper introduces the concept of B-Almost distributive fuzzy lattice (BADFL) in terms of its principal ideal fuzzy lattice. Necessary and sufficient conditions for an ADFL to become a B-ADFL are investigated. We also prove the equivalency of $B$-algebra and $B$-fuzzy algebra. In addition, we extend PSADL to PSADFL and prove that B-ADFL implies PSADFL.

Keywords: almost distributive lattice, Heyting almost distributive lattice (HADL), B-almost distributive lattice, principal ideals of an almost distributive lattice.
\end{abstract}

\section{Introduction}

The concept of an Almost distributive lattice was introduced by Swamy and Rao in [9]. It is an algebraic structure which satisfies almost all the properties of a distributive lattice with the smallest element except the commutativity of the operations $\wedge$ and $\vee$ and the right distributive of $\vee$ over $\wedge$. The concept of pseudo-complementation in an Almost distributive lattice was introduced by Swamy, Rao and Nanaji in [5] and the notion of Brikhoff center of an Almost distributive lattice with maximal element was introduced by Swamy and Ramesh in [8]. Heyting algebra is a distributive lattice in which, for any $a, b \in R$, the largest element $a \rightarrow b$ in $R$ exists with the property $a \wedge(a \rightarrow b) \leq b,[5]$. Heyting algebra serves as the algebraic models of propositional intutionistic logic in the same way as Boolean algebras model propositional classical logic. The concept of Heyting Almost distributive lattice (HADL) was introduced by Rao, Berhanu and Ratnamani in [5]. Studying the properties of Post algebra, G. Epstein and A. Horn, [2], introduced the concept of B-algebra as a bounded distributive lattice with center $B(R)$ in which for any $a, b \in R$, there exists the 
largest element $a \Rightarrow b$ in $B(R)$ exists with the property $a \wedge(a \Rightarrow b) \leq b$. In this paper,we introduce the concept of B-Almost distributive fuzzy lattice (B-ADFL) as an extension of B-ADL in which the fuzzy lattice of all principal ideals of $(R, A)$ is a B-fuzzy algebra. We also characterize necessary and sufficient conditions for an ADFL to become a B-ADFL. Throughout this paper $(R, A)$ represents an ADFL which again becomes B-ADFL. $B_{A}(R)$ represents a Birkhoff center of $(R, A)$ and $A: R \times R \rightarrow[0,1]$

\section{Preliminaries}

Definition 1. [7] An algebra $(R, \vee, \wedge, 0)$ of type $(2,2,0)$ is said to be an Almost distributive lattice (ADL) with 0 if it satisfies the following condition:

1. $a \vee 0=a$.

2. $0 \wedge a=0$.

3. $(a \vee b) \wedge c=(a \wedge c) \vee(b \wedge c)$.

4. $a \wedge(b \vee c)=(a \wedge b) \vee(a \wedge c)$.

5. $a \vee(b \wedge c)=(a \vee b) \wedge(a \vee c)$.

6. $(a \vee b) \wedge b=b$ for all $a, b, c \in R$.

Definition 2. [7] Let $\mathrm{X}$ be a non- empty set. Fix $x_{0} \in X$. For any $x, y \in X, x \wedge y=\left\{\begin{array}{ll}x_{o} & \text { if } x=x_{o} \\ y & \text { if } x \neq x_{o}\end{array}\right.$ and $x \vee y=\left\{\begin{array}{ll}y & \text { if } x=x_{o} \\ x & \text { if } x \neq x_{o}\end{array}\right.$.

Then $\left(X, \vee, \wedge, x_{o}\right)$ is an ADL with $x_{o}$ as its zero element. This ADL is called a discrete ADL.

Lemma 2.1. [7] For any $a, b \in R$ we have

1. $a \wedge 0=0$ and $0 \vee a=a$

2. $a \wedge a=a, a \vee a=a$

3. $(a \wedge b) \vee b=b, a \vee(b \wedge a)=a$ and $a \wedge(a \vee b)=a$

4. $a \wedge b=b \Leftrightarrow a \vee b=a$

5. $a \wedge b=a \Leftrightarrow a \vee b=b$

6. $a \wedge b \leq b$ and $a \leq a \vee b$

7. $a \wedge b=b \wedge a$, whenever $a \leq b$

8. $a \vee(b \vee a)=a \vee b$ 
Theorem 2.2. [7] For any $a, b \in R$ we have :

1. $(a \vee b) \wedge c=(a \wedge c) \vee(b \wedge c)$

2. $\wedge$ is associative in $R$

3. $a \wedge b \wedge c=b \wedge a \wedge c$

From the above it follows that for any $a \in R$ the set $\{a \wedge x \mid x \in R\}$ forms $a$ bounded distributive lattice. In particular, we have $((a \wedge b) \vee c) \wedge x=$ $((a \vee c) \wedge(b \vee c)) \wedge x$ for all $a, b, c, x \in R$. An element $m \in R$ is said to be maximal if $m \leq x$ implies $m=x$.

Lemma 2.3. [7] Let $R$ be an $A D L$ with 0 and $m \in R$. Then the following are equivalent:

1. $m$ is a maximal element with respect to a $\operatorname{poset}(R, \leq)$.

2. $m \vee x=m$ for all $x \in R$.

3. $m \wedge x=x$ for all $x \in R$.

Definition 3. [7] Let $R$ be an ADL. Then $R$ is said to be relatively complemented if for any $a, b \in R$ with $a \leq b$, there exists $x \in[a, b]$ such that $x \wedge y=a$ and $x \vee y=b$, for some $y \in[a, b]$.

THEOREM 2.4. [7] The following are equivalent for any ADL with 0:

1. $R$ is relatively complemented.

2. Given $x, y \in R$, there exists $a \in R$ such that $a \wedge x=0$ and $a \vee x=x \vee y$

3. For any $x \in R$, the interval $[0, x]$ is complemented.

TheOREm 2.5. [7] A relatively complemented ADL $R$ is associative.

Definition 4. [7] Let $R$ be an ADL and $a \in R$. Define $(a]=\{a \wedge x \mid x \in R\}$

Lemma 2.6. [7] For any $a, b \in R$, the following holds:

1. $(a] \cap(b]=(a \wedge b]=(b \wedge a]$.

2. $(a] \vee(b]=(a \vee b]=(b \vee a)$.

TheOREM 2.7. [7] For any $a, b \in R$ we have the following:

1. $(a]=\{a \wedge x \mid x \in R\}$.

2. $a \in(b] \Leftrightarrow b \wedge a=a \Leftrightarrow(a] \subseteq(b]$. 
Definition 5. [5] Let $(R, \vee, \wedge, 0, m)$ be an ADL with maximal element $\mathrm{m}$. Suppose $\rightarrow$ is a binary operation on $R$ satisfying the following condition:

1. $a \rightarrow a=m$.

2. $(a \rightarrow b) \wedge b=b$.

3. $a \wedge(a \rightarrow b)=a \wedge b \wedge m$.

4. $a \rightarrow(b \wedge c)=(a \rightarrow b) \wedge(a \rightarrow c)$.

5. $(a \vee b) \rightarrow c=(a \rightarrow c) \wedge(b \rightarrow c)$, for all $a, b, c \in R$.

Then $(R, \vee, \wedge, \rightarrow, 0, m)$ is called Heyting Almost Distributive Lattice $(H A D L)$.

Lemma 2.8. [5] Let $(R, \vee, \wedge, \rightarrow, 0, m)$ be an $H A D L, x, y, a \in R$ and $x \leq y$. Then the following conditions hold:

1. $a \rightarrow x \leq a \rightarrow y$.

2. $y \rightarrow a \leq x \rightarrow a$.

Definition 6. [3] Let $\mathrm{X}$ be a non-empty set. $(X, A)$ is called fuzzy partially ordered set if it satisfies the following:

1. $A(x, x)=1$. That is $\mathrm{A}$ is reflexive.

2. $A(x, y)>0$ and $A(y, x)>0$ implies that $x=y$. That is $\mathrm{A}$ is antisymmetric.

3. $A(x, z) \geq \operatorname{Sup}_{y \in X} \min [A(x, y), A(y, z)]>0$. That is $\mathrm{A}$ is transitive.

If $\mathrm{A}$ is a fuzzy partial order relation in a set $\mathrm{X}$, then $(X, A)$ is called a fuzzy partial order relation or fuzzy poset.

Definition 7 . [3] Let $(X, A)$ be a fuzzy poset. Then $(X, A)$ is a fuzzy lattice if and only if $x \vee y$ and $x \wedge y$ exist, for all $x, y \in X$.

Definition 8. [3] Let $(X, A)$ be a fuzzy lattice. Then $(X, A)$ is distributive if and only if $x \wedge(y \vee z)=(x \wedge y) \vee(x \wedge z)$ and $(x \vee y) \wedge(x \vee z)=$ $x \vee(y \wedge z)$, for all $x, y, z \in X$.

Definition 9. [4] Let $(X, A)$ be a fuzzy lattice and $Y \subseteq X$. Then $\mathrm{Y}$ is an ideal of $(X, A)$.

1. If $x \in X$ and $y \in Y$ and $A(x, y)>0$, then $x \in Y$.

2. If $x, y \in Y$, then $x \vee y \in Y$. 
DeFinition 10. [8] Let $R$ be an ADL with a maximal element $m$ and $B(R)=\{a \in R \mid a \wedge b=0$ and $a \vee b$ is maximal, for some $b \in R\}$. Then $(B(R), \vee, \wedge)$ is a relatively complemented ADL and is called Birkhoff center of $R$.

Definition 11. [2] Let $R$ be a distributive lattice with 0,1 , and let $B(R)$ be the Birkhoff center of $R$. If for any $a, b \in R$, there exists the greatest element $y \in B(R)$ such that $a \wedge y \leq b$, then $R$ is called a B-algebra .

The element $y$ is denoted by $a \Rightarrow b$.

Definition 12. [6] An ADL $R$ is called a B-ADL if its principal ideal lattice $P I(R)$ is a B-algebra.

TheOREm 2.9. [6] Let $R$ be an ADL with Birkhoff center $B(R)$. Then $R$ is a $B-A D L$ if and only if for any $a, b \in R$, there exists $y \in B(R)$ satisfying the following condition:

$R_{1}: b \wedge a \wedge y=a \wedge y$.

$R_{2}:$ If $z \in B(R)$ such that $b \wedge a \wedge z=a \wedge z$, then $y \wedge z=z$.

Definition 13. Let $R$ be an ADL with Birkhoff center $B(R)$. Then $R$ is called a dual B-ADL if for any $a, b \in R$, there exists $y \in B(R)$ satisfying the following:

1. $(a \vee y) \wedge b=b$

2. If $z \in B(R)$ such that $(a \vee z) \wedge b=b$, then $z \wedge y=y$.

Definition 14. [2] Let $R$ be a distributive lattice with 0,1 and let $B(R)$ be the Birkhoff center of $R$. If for any $a, b \in R$ there exists the least element $y \in B(R)$ such that $b \leq a \vee y$, then $R$ is called dual B-algebra.

The element $y$ is denoted by $a \Leftarrow b$.

Definition 15. [1] Let $(R, \vee, \wedge, 0)$ be an algebra of type $(2,2,0)$. $(R, A)$ is an Almost Distributive Fuzzy Lattice (ADFL) if the following conditions are satisfied:

1. $A(a, a \vee 0)=A(a \vee 0, a)=1$.

2. $A(0,0 \wedge a)=A(0 \wedge a, 0)=1$.

3. $A((a \vee b) \wedge c,(a \wedge c) \vee(b \wedge c))=A((a \wedge c) \vee(b \wedge c),(a \vee b) \wedge c)=1$.

4. $A(a \wedge(b \vee c),(a \wedge b) \vee(a \wedge c))=A((a \wedge b) \vee(a \wedge c), a \wedge(b \vee c))=1$. 
5. $A(a \vee(b \wedge c),(a \vee b) \wedge(a \vee c))=A((a \vee b) \wedge(a \vee c), a \vee(b \wedge c))=1$.

6. $A((a \vee b) \wedge b, b)=A(b,(a \vee b) \wedge b)=1$, for all $a, b, c \in R$.

Definition 16. [1] Let $(R, A)$ be an ADFL. Then for any $a, b \in R a \leq b$ if and only if $A(a, b)>0$.

\section{B-Almost Distributive Fuzzy Lattice $(B-A D F L)$}

Definition 17. Let $(R, A)$ be an ADFL with a maximal element $m$ and $B_{A}(R)=\{a \in R \mid A(a \wedge b, 0)>0$ and $A((a \vee b) \vee x, a \vee b)>0$ for some $b \in R$ and for all $x \in R\}$. Then $\left(B_{A}(R), \vee, \wedge\right)$ is a relatively complemented $\mathrm{ADFL}$ and $B_{A}(R)$ is called the Birkhoff center of an $\operatorname{ADFL}(R, A)$.

Throughout this section $R$ stands for a B-ADL $(R, \vee, \wedge, \Rightarrow, 0)$ with a maximal element $m$ and Birkhoff center $B(R)$.

Definition 18. Let $([0, m], A)$ be a distributive fuzzy lattice and for any $b \in[0, m], b \wedge m$ is complemented in $[0, m]$ whose complement is $(b \wedge m)^{\prime}$ such that $A\left((b \wedge m) \wedge(b \wedge m)^{\prime}, 0\right)>0$ and $A\left(m,(b \wedge m) \vee(b \wedge m)^{\prime}\right)>0$.

Definition 19. Let $(R, A)$ be a distributive fuzzy lattice with 0,1 , and let $B_{A}(R)$ be a Birkhoff center of $(R, A)$. If, for any $a, b \in R$, there exists the greatest element $y \in B_{A}(R)$ such that $A(a \wedge y, b)>0$, then $(R, A)$ is called a B-fuzzy algebra.

The element $y$ is denoted by $a \Rightarrow b$.

Lemma 3.1. Let $(R, A)$ be a distributive fuzzy lattice with 0,1 . Then $R$ is a B-algebra if and only if $(R, A)$ is a B-fuzzy algebra.

Proof. Assume that $R$ is a B-algebra. For any $a, b \in R$, there exist the greatest element $y \in B(R)$ such that $a \wedge y \leq b$. Let $(R, A)$ be an ADFL and let $B_{A}(R)$ be a Birkhoff center of $(R, A)$. Then, by assumption, for any $a, b \in(R, A)$, there exists $y \in B_{A}(R)$ such that $a \wedge y \leq b$. Hence $A(a \wedge y, b)>0$. Therefore, $(R, A)$ is a B-fuzzy algebra. Conversely, suppose that $(R, A)$ is a distributive fuzzy lattice with $0,1, B_{A}(R)$ is Birkhoff center of $(R, A)$ and $(R, A)$ is a B-fuzzy algebra. For any $a, b \in R$, there exists the greatest element $y \in B_{A}(R)$ such that $A(a \wedge y, b)>0$. So we get $a \wedge y \leq b$, which implies that $y \in B(R)$. Thus $R$ is a B-algebra. 
Definition 20. Let $(R, A)$ be an ADFL with maximal element. Then $(P I(R), A)$ is the set of all principal ideal fuzzy lattice of $(R, A)$, where $P I(R)$ is the set of principal ideal lattice of an $\operatorname{ADL}(R, \vee, \wedge, 0)$ with maximal element.

Definition 21. An ADFL $(R, A)$ is called a B-ADFL if its principal ideal fuzzy lattice $(P I(R), A)$ is a $\mathrm{B}$-fuzzy algebra.

In the following theorem we give an elementwise characterization for an ADFL to become a B-ADFL.

Theorem 3.2. Let $(R, A)$ be an ADFL with Birkhoff center $B_{A}(R)$. Then $(R, A)$ is a $B$-ADFL if and only if for any $a, b \in R$, there exists $y \in B_{A}(R)$ satisfying the following conditions:

$L_{1}: A(a \wedge y, b \wedge a \wedge y)>0$.

$L_{2}:$ If $z \in B_{A}(R)$ such that $A(a \wedge z, b \wedge a \wedge z)>0$, then $A(z, y \wedge z)>0$.

Proof. Suppose that $(R, A)$ is a B-ADFL and $a, b \in R$. Then $(P I(R), A)$ is a B-fuzzy algebra, so $(a]_{A} \Rightarrow(b]_{A}=(y]_{A}$ for some $y \in B_{A}(R)$. Now, $(a]_{A} \cap(y]_{A} \subseteq(b]_{A} \Leftrightarrow(a \wedge y]_{A} \subseteq(b]_{A}$ and hence $a \wedge y \in(b]_{A}$, which implies $A(a \wedge y, b \wedge a \wedge y)>0$. Now, $(b \wedge a \wedge y) \wedge a \wedge y=b \wedge((a \wedge y) \wedge(a \wedge y)=b \wedge a \wedge y$ by idempotence and associativity of $\wedge$. $(b \wedge a \wedge y) \vee(a \wedge y)=a \wedge y$ by the absorption property. Hence $b \wedge a \wedge y \leq a \wedge y$, we get $A(b \wedge a \wedge y, a \wedge y)>0$. So we have $b \wedge a \wedge y=a \wedge y$ by the antisymmetry property of $A$. Therefore, $A(a \wedge y, b \wedge a \wedge y)>0$. Let $z \in B_{A}(R)$ such that $A(a \wedge z, b \wedge a \wedge z)>0$. Then $(a]_{A} \cap(z]_{A} \subseteq(b]_{A} \Leftrightarrow(a \wedge z]_{A} \subseteq(b]_{A} \Rightarrow a \wedge z \in(b]_{A}$. Hence $A(a \wedge z, b \wedge a \wedge z)>$ 0 . Since $b \wedge a \wedge z \leq a \wedge z$, we have $A(b \wedge a \wedge z, a \wedge z)>0$. Hence $b \wedge a \wedge z=a \wedge z$ by antisymmetry of $A$ and we have $A(a \wedge z, b \wedge a \wedge z)>0$. Consequently, $(z]_{A} \subseteq(y]_{A}$, which implies that $z \in(y]_{A}$. Hence $A(z, y \wedge z)>0$ and since $y \wedge z \leq z$, we get $A(y \wedge z, z)>0$, so that we have $y \wedge z=z$ by antisymmetry of $A$. Therefore $A(z, y \wedge z)>0$. Conversely, suppose that $(R, A)$ satisfies $L_{1}$ and $L_{2}$, and let $(a],(b] \in(P I(R), A)$, where $a, b \in R$. Then there exists $y \in B_{A}(P I(R))$ satisfying $L_{1}$ and $L_{2}$. Again, $(y] \in B_{A}(P I(R))$ and $A(a \wedge y, b \wedge a \wedge y)>0$ implies that $(a]_{A} \cap(y]_{A} \subseteq(b]_{A} \Leftrightarrow(a \wedge y]_{A} \subseteq(b]_{A}$ and hence $a \wedge y \in(b]_{A}$. Therefore, $A(a \wedge y, b \wedge a \wedge y)>0$. Since $b \wedge(a \wedge y) \leq a \wedge y$, we have $A(b \wedge a \wedge y, a \wedge y)>0$. Therefore, $b \wedge a \wedge y=a \wedge y$ by antisymmetry of $A$. Hence $A(a \wedge y, b \wedge a \wedge y)>0$, which implies that $(a]_{A} \cap(y]_{A} \subseteq(b]_{A}$. We know that $(z] \in B_{A}(P I(R))$ such that $(a]_{A} \cap(z]_{A} \subseteq(b]_{A} \Leftrightarrow(a \wedge z]_{A} \subseteq(b]_{A}$ implies that $a \wedge z \in(b]_{A}$. Hence $A(a \wedge z, b \wedge a \wedge z)>0$. Since $b \wedge a \wedge z \leq a \wedge z$, 
we have $A(b \wedge a \wedge z, a \wedge z)>0$. Therefore, $b \wedge a \wedge z=a \wedge z$ implies that $A(a \wedge z, b \wedge a \wedge z)>0$. So $(z]_{A} \subseteq(y]_{A}$ implies that $z \in(y]_{A}$ and hence $A(z, y \wedge z)>0$ and we get $(z]_{A} \subseteq(y]_{A}$. Therefore, $(P I(R), A)$ is a B-fuzzy algebra. Consequently, $(R, A)$ is a B-ADFL. From Theorem 3.2 we derive the following corollaries.

Corollary 3.3. Let $(R, A)$ be a $B$-ADFL with a maximal element $m$ and Birkhoff center $B_{A}(R)$. If, for any $a, b \in R$, there exist $y, z \in B_{A}(R)$ satisfying $L_{1}$ and $L_{2}$ of Theorem 3.2, then $A(y \wedge m, z \wedge m)=A(z \wedge m, y \wedge$ $m)=1$.

Proof. Let $a, b \in R$ and $y, z \in B_{A}(R)$ such that

$L_{1}: A(a \wedge y, b \wedge a \wedge y)>0$.

$L_{2}:$ If $z \in B_{A}(R)$ such that $A(a \wedge z, b \wedge a \wedge z)>0$, then $A(z, y \wedge z)>0$.

Now, $A(z \wedge m, y \wedge m)=A((a \Rightarrow b) \wedge m,(a \Rightarrow b) \wedge m)=A\left(a \Rightarrow_{m} b, a \Rightarrow_{m}\right.$ $b)=1$. Hence $A(z \wedge m, y \wedge m)=1$. Similarly, $A(y \wedge m, z \wedge m)=1$. Therefore, $A(z \wedge m, y \wedge m)=A(y \wedge m, z \wedge m)=1$. Here afterwards $(R, A)$ stands a B-ADFL with maximal element $m$ and Birkhoff center $B_{A}(R)$.

Definition 22. Let $a, b \in(R, A)$. If $y \in B_{A}(R)$ satisfies $L_{1}$ and $L_{2}$ of Theorem 3.2 , then we denote $y \wedge m$ by $a \Rightarrow_{m} b$ or simply $a \Rightarrow b$, where there is no ambiguity.

Corollary 3.4. For any $a, b \in R$, we have the following:

1. $A(b \wedge a \wedge(a \Rightarrow b), a \wedge(a \Rightarrow b))=A(a \wedge(a \Rightarrow b), b \wedge a \wedge(a \Rightarrow b))=1$ and, consequently, $A(a \wedge(a \Rightarrow b), b \wedge m)>0$.

2. If $z \in B_{A}(R), A(a \wedge z, b \wedge m)>0$, then $A(z \wedge m, a \Rightarrow b)>0$.

3. $A(a \wedge m, b \wedge m)>0$ if and only if $A(a \Rightarrow b, m)=A(m, a \Rightarrow b)=1$.

Corollary 3.5. For any $a, b \in R$, we have the following:

1. $A(0 \Rightarrow a, m)=A(m, 0 \Rightarrow a)=1$.

2. $A(a \Rightarrow a, m)=A(m, a \Rightarrow a)=1$.

3. $A(a \Rightarrow m, m)=A(m, a \Rightarrow m)=1$.

4. $A(a \wedge b) \Rightarrow a, m)=A(m,(a \wedge b) \Rightarrow a)=1$. and $A((a \wedge b) \Rightarrow b, m)=$ $A(m,(a \wedge b) \Rightarrow b)=1$.

5. $A(a \Rightarrow(a \vee b), m)=A(m, a \Rightarrow(a \vee b))=1$ and $A(a \Rightarrow(b \vee a), m)=$ $A(m, a \Rightarrow(b \vee a))=1$.

6. $A((a \wedge b) \Rightarrow(a \vee b), m)=A(m,(a \wedge b) \Rightarrow(a \vee b))=1$. 
Theorem 3.6. Let $(R, A)$ be a $B$-ADFL. If $a \in R$ and $b \in B_{A}(R)$, then the following hold:

1. $A(a \wedge(a \Rightarrow b), a \wedge b \wedge m)=A(a \wedge b \wedge m, a \wedge(a \Rightarrow b))=1$.

2. $A(a \wedge(a \Rightarrow(b \Rightarrow c), a \wedge(b \Rightarrow c))=A(a \wedge(b \Rightarrow c), a \wedge(a \Rightarrow(b \Rightarrow c))=1$.

3. $A(b \wedge(a \Rightarrow b), b \wedge m)=A(b \wedge m, b \wedge(a \Rightarrow b))=1$.

4. $A((a \Rightarrow b) \wedge b, b)=A(b,(a \Rightarrow b) \wedge b)=1$.

Proof. Let $a, b \in R$.

1. By Corollary $3.4 A(a \wedge(a \Rightarrow b), b \wedge m)>0$, so $A(a \wedge(a \Rightarrow b), a \wedge b \wedge m)>$ 0 . Since $b \in B_{A}(R)$ and $A(a \wedge b \wedge m, b \wedge m)>0$ by Corollary 3.4 again, $A(b \wedge m, a \Rightarrow b)>0$ and hence $A(a \wedge b \wedge m, a \wedge(a \Rightarrow b))>0$. Therefore, $a \wedge(a \Rightarrow b)=a \wedge b \wedge m$ by antisymmetry property of $\mathrm{A}$. Hence $A(a \wedge(a \Rightarrow b), a \wedge b \wedge m)=A(a \wedge b \wedge m, a \wedge(a \Rightarrow b))=1$.

2. By Corollary 3.4 we have $A(m, b \Rightarrow c)=A(b \Rightarrow c, m)=1$. Then $A(a \wedge(a \Rightarrow(b \Rightarrow c), a \wedge(b \Rightarrow c))=A(a \wedge(a \Rightarrow m), a \wedge m)=A(a \wedge$ $m, a \wedge m)=1$ since $a \Rightarrow m=m$. Hence $A(a \wedge(a \Rightarrow(b \Rightarrow c), a \wedge(b \Rightarrow$ $c))=1$. Similarly, $A(a \wedge(b \Rightarrow c), a \wedge(a \Rightarrow(b \Rightarrow c))=1$. Therefore $A(a \wedge(a \Rightarrow(b \Rightarrow c), a \wedge(b \Rightarrow c))=A(a \wedge(b \Rightarrow c), a \wedge(a \Rightarrow(b \Rightarrow c))=1$.

3. Assume that $A(b \wedge m, a \Rightarrow b)>0$. We get $A(b \wedge m, b \wedge(a \Rightarrow b))>$ 0 , since $b \wedge m=b \wedge m \wedge(a \Rightarrow b)=b \wedge(a \Rightarrow b)$. Similarly, $A(b \wedge(a \Rightarrow$ $b), b \wedge m)>0$. Thus, $A(b \wedge(a \Rightarrow b), b \wedge m)=A(b \wedge m, b \wedge(a \Rightarrow b))=1$.

4. Now, $A(b,(a \Rightarrow b) \wedge b)=A(b \wedge m \wedge b,(a \Rightarrow b) \wedge b)$ since $b=b \wedge m \wedge b$. $=$ $A(b \wedge(a \Rightarrow b) \wedge b,(a \Rightarrow b) \wedge b)=A((a \Rightarrow b) \wedge b,(a \Rightarrow b) \wedge b)=1$. Hence $A(b,(a \Rightarrow b) \wedge b)=1$. Similarly $A((a \Rightarrow b) \wedge b, b)=1$. Therefore, $A(b,(a \Rightarrow b) \wedge b)=A((a \Rightarrow b) \wedge b, b)=1$.

TheOrem 3.7. Let $a, b, c \in R$ be any element and let $m$ be a maximal element of $R$. Then the following hold:

1. If $A(a \wedge m, b \wedge m)>0$, then $A(c \Rightarrow a, c \Rightarrow b)>0$ and $A(b \Rightarrow c, a \Rightarrow$ c) $>0$.

2. $A(c \Rightarrow(a \wedge b),(c \Rightarrow a) \wedge(c \Rightarrow b))=$ $=A((c \Rightarrow a) \wedge(c \Rightarrow b), c \Rightarrow(a \wedge b))=A(c \Rightarrow(a \wedge b), c \Rightarrow(b \wedge a))=$ $A(c \Rightarrow(b \wedge a), c \Rightarrow(a \wedge b))=1$.

3. $A((a \vee b) \Rightarrow c,(a \Rightarrow c) \wedge(b \Rightarrow c))=A((a \Rightarrow c) \wedge(b \Rightarrow c),(a \vee b) \Rightarrow c)$ $=A((a \vee b) \Rightarrow c,(b \vee a) \Rightarrow c)=A((b \vee a) \Rightarrow c,(a \vee b) \Rightarrow c)=1$.

4. $A(c \Rightarrow(a \vee b), c \Rightarrow(b \vee a))=A(c \Rightarrow(b \vee a), c \Rightarrow(a \vee b))=1$.

5. $A((a \wedge b) \Rightarrow c,(b \wedge a) \Rightarrow c)=A((b \wedge a) \Rightarrow c,(a \wedge b) \Rightarrow c)=1$. 
Proof. Let $a, b, c \in R$.

1. If $A(a \wedge m, b \wedge m)>0$, then $A(c \wedge(c \Rightarrow a), a \wedge m)>0$ and $A(a \wedge m, b \wedge$ $m)>0, A(c \wedge(c \Rightarrow a), b \wedge m)>0$. So we get $A(c \Rightarrow a, c \Rightarrow b)>0$. Also we have $A(a \wedge(b \Rightarrow c), b \wedge(b \Rightarrow c))>0$, take $m=b \Rightarrow c$. $A(b \wedge(b \Rightarrow c), c \wedge m)>0, A(a \wedge(b \Rightarrow c), c \wedge m)>0$ and hence $A(b \Rightarrow c, a \Rightarrow c)>0$.

2. From (1), we get $A(c \Rightarrow(a \wedge b),(c \Rightarrow a) \wedge(c \Rightarrow b))>0$ since $a \wedge b \leq b$ imply that $c \Rightarrow(a \wedge b) \leq c \Rightarrow a, c \Rightarrow(a \wedge b) \leq c \Rightarrow b$. On the other hand, we have $A(c \wedge(c \Rightarrow a) \wedge(c \Rightarrow b), a \wedge b \wedge m)>0$. So $A((c \Rightarrow$ $a) \wedge(c \Rightarrow b), c \Rightarrow(a \wedge b))>0$. Hence $c \Rightarrow(a \wedge b)=(c \Rightarrow a) \wedge(c \Rightarrow b)$ by antisymmetry property of $\mathrm{A}$. Therefore, $A(c \Rightarrow(a \wedge b),(c \Rightarrow a) \wedge(c \Rightarrow$ $b))=A((c \Rightarrow a) \wedge(c \Rightarrow b), c \Rightarrow(a \wedge b))=1$.

3. Assume that $A(a \wedge m,(a \vee b) \wedge m)>0$ and $A(b \wedge m,(a \vee b) \wedge m)>0$, by (1) above $A((a \vee b) \Rightarrow c,(a \Rightarrow c) \wedge(b \Rightarrow c))>0$, since $A((a \vee b) \Rightarrow$ $c, a \Rightarrow c)>0$, and $A((a \vee b) \Rightarrow c, b \Rightarrow c)>0$. On the other hand, $A((a \vee b) \wedge(a \Rightarrow c) \wedge(b \Rightarrow c), c \wedge m)>0$ and hence $A((a \Rightarrow c) \wedge(b \Rightarrow$ $c),(a \vee b) \Rightarrow c)>0$. Hence $(a \vee b) \Rightarrow c=(a \Rightarrow c) \wedge(b \Rightarrow c)$ by antisymmetry of $A$. Therefore, $A((a \vee b) \Rightarrow c,(a \Rightarrow c) \wedge(b \Rightarrow c))=$ $A((a \Rightarrow c) \wedge(b \Rightarrow c),(a \vee b) \Rightarrow c)=1$.

4. Assume that $A((a \vee b) \wedge c,(b \vee a) \wedge c)=A((b \vee a) \wedge c,(a \vee b) \wedge c)=1$ and $A(c \wedge(c \Rightarrow(a \vee b)),(a \vee b) \wedge m)=A(c \wedge(c \Rightarrow(a \vee b)),(b \vee a) \wedge m)=A((a \vee$ $b) \wedge m,(c \wedge(c \Rightarrow(a \vee b)))>0$. We get $A(c \Rightarrow(a \vee b), c \Rightarrow(b \vee a))>0$. Interchanging $a$ and $b$, we have $A(c \wedge(c \Rightarrow(b \vee a)),(b \vee a) \wedge m)=$ $A(c \wedge(c \Rightarrow(b \vee a)),(a \vee b) \wedge m)=A((b \vee a) \wedge m, c \wedge(c \Rightarrow(b \vee a)))>0$. We get $A(c \Rightarrow(b \vee a), c \Rightarrow(a \vee b))>0$ and hence $c \Rightarrow(a \vee b)=c \Rightarrow(b \vee a)$ by antisymmetry of $A$. Therefore, $A(c \Rightarrow(a \vee b), c \Rightarrow(b \vee a))=A(c \Rightarrow$ $(b \vee a), c \Rightarrow(a \vee b))=1$.

5. Suppose that $A(a \wedge b \wedge c, b \wedge a \wedge c)=A(b \wedge a \wedge c, a \wedge b \wedge c)=1$ and $A((a \wedge b) \wedge((b \wedge a) \Rightarrow c),(b \wedge a) \wedge((b \wedge a) \Rightarrow c))=A((b \wedge a) \wedge((b \wedge a) \Rightarrow$ $c),(a \wedge b) \wedge((b \wedge a) \Rightarrow c)=1$. Also, $A((b \wedge a) \wedge((b \wedge a) \Rightarrow c), c \wedge m)>0$ and $A((a \wedge b) \wedge((b \wedge a) \Rightarrow c), c \wedge m)>0$. Since $(a \wedge b) \wedge((b \wedge a) \Rightarrow c)=$ $(b \wedge a) \wedge((b \wedge a) \Rightarrow c) \leq c \wedge m$, then $A((b \wedge a) \Rightarrow c,(a \wedge b) \Rightarrow c)>0(*)$. Interchanging $a$ and $b$, we get $A((a \wedge b) \Rightarrow c,(b \wedge a) \Rightarrow c)>0$ (**). Hence $(a \wedge b) \Rightarrow c=(b \wedge a) \Rightarrow c$ by antisymmetry of $A$. Therefore, $A((a \wedge b) \Rightarrow c,(b \wedge a) \Rightarrow c)=A((b \wedge a) \Rightarrow c,(a \wedge b) \Rightarrow c)=1$. 
Corollary 3.8. For any $a, b \in R$ the following hold:

1. $A(a \Rightarrow(a \wedge b), a \Rightarrow(b \wedge a))=A(a \Rightarrow(b \wedge a), a \Rightarrow(a \wedge b))$

$=A(a \Rightarrow(a \wedge b), a \Rightarrow b)=A(a \Rightarrow b, a \Rightarrow(a \wedge b))$

$=A(a \Rightarrow b, a \Rightarrow(b \wedge a))=A(a \Rightarrow(b \wedge a), a \Rightarrow b)=1$.

2. $A(a \Rightarrow(b \wedge m), a \Rightarrow b)=A(a \Rightarrow b, a \Rightarrow(b \wedge m))=1$.

3. $A((a \vee b) \Rightarrow b,(b \vee a) \Rightarrow b)=A((b \vee a) \Rightarrow b,(a \vee b) \Rightarrow b)$

$=A((a \vee b) \Rightarrow b, a \Rightarrow b)=A(a \Rightarrow b,(a \vee b) \Rightarrow b)$

$=A(a \Rightarrow b,(b \vee a) \Rightarrow b)=A((b \vee a) \Rightarrow b, a \Rightarrow b)=1$.

4. $A((a \vee b) \Rightarrow(a \wedge b),(b \Rightarrow a) \wedge(a \Rightarrow b))$ $=A((b \Rightarrow a) \wedge(a \Rightarrow b),(a \vee b) \Rightarrow(a \wedge b))=1$.

5. $A((a \wedge m) \Rightarrow b, a \Rightarrow b)=A(a \Rightarrow b,(a \wedge m) \Rightarrow b)=1$.

6. $A((a \wedge m) \Rightarrow(b \wedge m), a \Rightarrow b)=A(a \Rightarrow b,(a \wedge m) \Rightarrow(b \wedge m))=1$.

Proof. Let $a, b \in R$.

1. $A(a \Rightarrow(a \wedge b), a \Rightarrow b)=A((a \Rightarrow a) \wedge(a \Rightarrow b), a \Rightarrow b)=A(m \wedge(a \Rightarrow$ $b), a \Rightarrow b)=A(a \Rightarrow b, a \Rightarrow b)=1$ since $a \Rightarrow a=m$ and $m \wedge$ $(a \Rightarrow b)=a \Rightarrow b$. Hence $A(a \Rightarrow(a \wedge b), a \Rightarrow b)=1$. Similarly, $A(a \Rightarrow b, a \Rightarrow(a \wedge b))=1$. Therefore, $A(a \Rightarrow(a \wedge b), a \Rightarrow b)$ $=A(a \Rightarrow b, a \Rightarrow(a \wedge b))=1 . \quad A(a \Rightarrow(b \wedge a), a \Rightarrow b)=A((a \Rightarrow$ b) $\wedge(a \Rightarrow a), a \Rightarrow b)=A((a \Rightarrow b) \wedge m, a \Rightarrow b)$, since $a \Rightarrow a=m$ and $m \leq a \Rightarrow b . A(m \wedge(a \Rightarrow b), a \Rightarrow b)=A(a \Rightarrow b, a \Rightarrow b)=1$, since $m \wedge(a \Rightarrow b)=a \Rightarrow b$. Hence $A(a \Rightarrow(b \wedge a), a \Rightarrow b)=1$. Similarly, $A(a \Rightarrow b, a \Rightarrow(b \wedge a))=1$. Therefore, $A(a \Rightarrow(b \wedge a), a \Rightarrow b)=A(a \Rightarrow$ $b, a \Rightarrow(b \wedge a))=1$. Thus, $A(a \Rightarrow(a \wedge b), a \Rightarrow(b \wedge a)=A(a \Rightarrow$ $(b \wedge a), a \Rightarrow(a \wedge b))=1$.

2. $A(a \Rightarrow(b \wedge m), a \Rightarrow b)=A((a \Rightarrow b) \wedge(a \Rightarrow m), a \Rightarrow b)$. Ву 1, $A(a \Rightarrow b, a \Rightarrow b)=1$, since $m \wedge(a \Rightarrow b)=a \Rightarrow b$. Hence $A(a \Rightarrow(b \wedge m), a \Rightarrow b)=1$. Similarly, $A(a \Rightarrow b, a \Rightarrow(b \wedge m))=1$. Therefore, $A(a \Rightarrow(b \wedge a), a \Rightarrow b)=A(a \Rightarrow b, a \Rightarrow(b \wedge a))=1$.

3. $A((a \vee b) \Rightarrow b, a \Rightarrow b)=A((a \Rightarrow b) \wedge(b \Rightarrow b), a \Rightarrow b)=A((a \Rightarrow b) \wedge$ $m, a \Rightarrow b)$, since $b \Rightarrow b=m=A(m \wedge(a \Rightarrow b), a \Rightarrow b)=A(a \Rightarrow b, a \Rightarrow$ $b)=1$, since $m \wedge(a \Rightarrow b)=a \Rightarrow b$. Hence $A((a \vee b) \Rightarrow b, a \Rightarrow b)=1$. Similarly, $A(a \Rightarrow b,(a \vee b) \Rightarrow b)=1$. Therefore, $A((a \vee b) \Rightarrow b, a \Rightarrow$ $b)=A(a \Rightarrow b,(a \vee b) \Rightarrow b)=1$. Again, $A((b \vee a) \Rightarrow a, a \Rightarrow b)=$ $A((b \Rightarrow a) \wedge(a \Rightarrow a), a \Rightarrow b)=A((b \Rightarrow a) \wedge m, a \Rightarrow b)$, since $a \Rightarrow$ $a=m=A(m \wedge(b \Rightarrow a), a \Rightarrow b)=A(b \Rightarrow a, a \Rightarrow b)=A(a \Rightarrow b . a \Rightarrow$ $b)=1$ since $(a \vee b) \Rightarrow b=(b \vee a) \Rightarrow a)$ implies that $b \Rightarrow a=a \Rightarrow b$. 
Hence $A((b \vee a) \Rightarrow a, a \Rightarrow b)=1$. Similarly, $A(a \Rightarrow b,(b \vee a) \Rightarrow a)=1$. Therefore, $A((b \vee a) \Rightarrow a, a \Rightarrow b)=A(a \Rightarrow b,(b \vee a) \Rightarrow a)=1$.

4. $A((a \vee b) \Rightarrow(b \wedge a),(a \Rightarrow b) \wedge(b \Rightarrow a))=A([(a \vee b) \Rightarrow b] \wedge[(a \vee b) \Rightarrow$ $a],(a \Rightarrow b) \wedge(b \Rightarrow a))=A([(a \Rightarrow b) \wedge(b \Rightarrow b)] \wedge[(a \Rightarrow a) \wedge(b \Rightarrow a)],(a \Rightarrow$ $b) \wedge(b \Rightarrow a))=A([(a \Rightarrow b) \wedge m] \wedge[m \wedge(b \Rightarrow a)],(a \Rightarrow b) \wedge(b \Rightarrow a))$, since $a \Rightarrow a=m, b \Rightarrow b=m A(m \wedge(a \Rightarrow b), m \wedge(b \Rightarrow a))=A((a \Rightarrow b) \wedge(b \Rightarrow$ $a),(a \Rightarrow b) \wedge(b \Rightarrow a))=A((a \Rightarrow b) \wedge(b \Rightarrow a),(a \Rightarrow b) \wedge(b \Rightarrow a))=1$. Hence $A((a \vee b) \Rightarrow(a \wedge b),(a \Rightarrow b) \wedge(b \Rightarrow a))=1$. Similarly, $A((a \Rightarrow b) \wedge(b \Rightarrow a),(a \vee b) \Rightarrow(a \wedge b))=1$. Therefore, $A((a \vee b) \Rightarrow$ $(a \wedge b),(a \Rightarrow b) \wedge(b \Rightarrow a))=A((a \Rightarrow b) \wedge(b \Rightarrow a),(a \vee b) \Rightarrow(a \wedge b))=1$.

5. $A((a \wedge m) \Rightarrow b, a \Rightarrow b)=A((m \wedge a) \Rightarrow b, a \Rightarrow b)=A(a \Rightarrow b, a \Rightarrow b)=1$ since $m \wedge a=a$. Hence $A((a \wedge m) \Rightarrow b, a \Rightarrow b)=1$. Similarly, $A(a \Rightarrow b,(a \wedge m) \Rightarrow b)=1$. Therefore, $A((a \wedge m) \Rightarrow b, a \Rightarrow b)=$ $A(a \Rightarrow b,(a \wedge m) \Rightarrow b)=1$.

6. $A((a \wedge m) \Rightarrow(b \wedge m), a \Rightarrow b)=A((m \wedge a) \Rightarrow(m \wedge b), a \Rightarrow b)=A(a \Rightarrow$ $b, a \Rightarrow b)=1$. Since $m \wedge a=a, m \wedge b=b$, then $A((a \wedge m) \Rightarrow$ $(b \wedge m), a \Rightarrow b)=1$. Similarly, $A(a \Rightarrow b,(a \wedge m) \Rightarrow(b \wedge m))=1$. Therefore, $A((a \wedge m) \Rightarrow(b \wedge m), a \Rightarrow b)=A(a \Rightarrow b,(a \wedge m) \Rightarrow(b \wedge m))=1$.

Definition 23. Let $(R, A)$ be an ADFL with a maximal element $m$ and Birkhoff center $B_{A}(R)$. Then $(R, A)$ is called Pseudo-supplemented Almost Distributive Fuzzy Lattice $(P S A D F L)$ if, for each $a \in R$, there exists $y \in B_{A}(R)$ such that

$P_{1}: A(y, a \wedge y)>0$.

$P_{2}:$ If $z \in B_{A}(R)$ and $A(z, a \wedge z)>0$, then $A(z, y \wedge z)>0$.

Now, we prove that every B-ADFL is a PSADFL in the following theorem.

Theorem 3.9. Let $(R, A)$ be a $B$-ADFL with a maximal element $m$ and Birkhoff center $B_{A}(R)$. Then $(R, A)$ is a PSADFL.

Proof. Suppose that $(R, A)$ is a B-ADFL and $a \in R$. Then we have $A(y, m \Rightarrow a)=A(m \Rightarrow a, y)=1$. Then $y \in B_{A}(R)$ and $A(y, a \wedge m)>0$. Now, $A(y, a \wedge y)=A(y \wedge y, a \wedge y)=A(y \wedge a \wedge m \wedge y, a \wedge y)=A(y \wedge a \wedge y, a \wedge y)$, since $m \wedge y=y=A(a \wedge y, a \wedge y)=1$ since $m \wedge a=a$. Hence $A(y, a \wedge y)=1$. Similarly, $A(a \wedge y, y)=1$. Therefore, $A(y, a \wedge y)=A(a \wedge y, y)=1$. So we get $A(y, a \wedge y)>0$. 
(2) Suppose that $z \in B_{A}(R)$ such that $A(z, a \wedge z)>0$. Then $A(z \wedge m, a \wedge$ $m)>0$ and hence $A(z \wedge m, m \Rightarrow a=y)>0 . A(z, y \wedge z)=A(z \wedge m \wedge z, y \wedge z)$ $=A(z \wedge m \wedge a \wedge z, y \wedge z)$ since $z \wedge m=a \wedge m \wedge z \wedge m$. $=A(y \wedge z \wedge m, y \wedge z)$ $=A(y \wedge z, y \wedge z)=1$. Hence $A(z, y \wedge z)=1$. Similarly, $A(y \wedge z, z)=1$. Therefore, $A(z, y \wedge z)=A(y \wedge z, z)=1)$. We get $A(z, y \wedge z)>0$ and $A(y \wedge z, z)>0$. So we have $z=y \wedge z$. Therefore, $A(z, y \wedge z)>0$. Thus, $(R, A)$ is a PSADFL. The following Lemma can be proved easily since for a maximal element $m$ the interval $([0, m], A)$ is a distributive fuzzy lattice.

TheOREm 3.10. Let $(R, A)$ be an ADFL with a maximal element $m$ and Birkhoff center $B_{A}(R)$. Then, for any $a, b \in R$ and $y \in B_{A}(R)$ :

1. $A\left(b \wedge m,\left[(y \wedge m)^{\prime} \vee a\right] \wedge m\right)>0$ if and only if $A((y \wedge b) \wedge m, a \wedge m)>0$.

2. $A\left((y \wedge m)^{\prime} \wedge a \wedge m, y \wedge m\right)>0$ if and only if $A(a \wedge m .(y \vee b) \wedge m)>0$, where $(y \wedge m)^{\prime}$ is the complement of $y \wedge m$ in $[0, m]$.

\section{PROOF.}

1. Suppose that $A\left(b \wedge m,\left[(y \wedge m)^{\prime} \vee a\right] \wedge m\right)>0$. Then

$$
\begin{aligned}
& \left.A\left(y \wedge m \wedge b \wedge m,(y \wedge m) \wedge(y \wedge m)^{\prime} \vee a\right] \wedge m\right) \\
= & A\left(y \wedge m \wedge b \wedge m,\left[(y \wedge m) \wedge(y \wedge m)^{\prime}\right] \vee[(y \wedge m) \wedge a] \wedge m\right) \\
= & A(y \wedge m \wedge b \wedge m, 0 \vee[(y \wedge m) \wedge a] \wedge m) \\
= & A(y \wedge m \wedge b \wedge m,(y \wedge a) \wedge m) \\
= & A(y \wedge b \wedge m,(y \wedge a) \wedge m) \\
= & A(y \wedge b \wedge m, y \wedge(a \wedge m)) \\
= & A(y, y)=1>0,
\end{aligned}
$$

since $y \wedge b \wedge m=y$ and $y \wedge a \wedge m=y$. As $A(y \wedge(a \wedge m), a \wedge m)>0$, we have $A(y \wedge b \wedge m, a \wedge m)>0 y \wedge a \wedge m=y \wedge b \wedge m$.

Conversely, suppose that $A(y \wedge b \wedge m, a \wedge m)>0$.

$$
\begin{aligned}
& A\left(m \wedge b,\left((y \wedge m)^{\prime} \vee a\right) \wedge m\right. \\
\geq & \left.(y \wedge m)^{\prime} \vee(y \wedge b \wedge m)\right) \\
= & \left.A\left(m \wedge b,(y \wedge m)^{\prime} \vee y \wedge m\right) \wedge\left((y \wedge m)^{\prime} \vee b\right)\right) \\
= & A\left(m \wedge b, m \wedge\left((y \wedge m)^{\prime} \vee b\right)\right) \\
= & A\left(m \wedge b,\left(m \wedge(y \wedge m)^{\prime} \vee(m \wedge b)\right)\right. \\
\geq & A(m \wedge b, m \wedge b)=1 .
\end{aligned}
$$


It implies that $A\left(b \wedge m,\left((y \wedge m)^{\prime} \vee a\right) \wedge m\right)=1$. Hence $A(b \wedge m,((y \wedge$ $\left.\left.m)^{\prime} \vee a\right) \wedge m\right)>0$.

2. Suppose that $A\left((y \wedge m)^{\prime} \wedge a \wedge m, y \wedge m\right)>0$.

$$
\begin{aligned}
A(a \wedge m,(y \vee b) \wedge m)= & A(a \wedge m,((y \wedge m) \vee(b \wedge m) \\
\geq & \left.\left.(y \wedge m) \vee\left((y \wedge m)^{\prime} \wedge a \wedge m\right)\right)\right) \\
= & A\left(a \wedge m,(y \wedge m) \vee(y \wedge m)^{\prime}\right. \\
& \wedge[(y \wedge m) \vee(a \wedge m)]) \\
= & A(a \wedge m, m \wedge(y \wedge m) \vee(a \wedge m)]) \\
= & A(a \wedge m, m \wedge[(y \vee a) \wedge m]) \\
\geq & A(a \wedge m, a \wedge m)=1 .
\end{aligned}
$$

So that $A(a \wedge m,(y \vee b) \wedge m)=1$ Hence $A(a \wedge m,(y \vee b) \wedge m)>0$. Conversely, suppose $A(a \wedge m,(y \vee b) \wedge m)>0$. Then $A\left((y \wedge m)^{\prime} \wedge a \wedge m, b \wedge m\right)>0$ since

$$
\begin{aligned}
A\left(b \wedge m,(y \wedge m)^{\prime} \wedge a \wedge m\right) \leq & A\left(b \wedge m,(y \wedge m)^{\prime} \wedge((y \vee b) \wedge m)\right) \\
= & A\left(b \wedge m,\left[(y \wedge m)^{\prime} \wedge y\right)\right. \\
& \left.\left.\vee\left((y \wedge m)^{\prime} \wedge b\right)\right] \wedge m\right) \\
= & A\left(b \wedge m,\left((y \wedge m)^{\prime} \wedge b\right) \wedge m\right) \\
\leq & A(b \wedge m, b \wedge m)=1 .
\end{aligned}
$$

\section{Coclusion}

In this paper we have extended B-ADL to B-ADFL. In a distributive fuzzy lattice with 0 and $1, B-$ algebra and $B-f u z z y$ algebra are equivalent. B-ADFL with a maximal element $m$ and Birkhoff center $B_{A}(R)$ implies PSADFL. In general, it is also posible to extend B-ADFL to dual B-ADFL.

\section{References}

[1] A. Berhanu, G. Yohannes and T. Bekalu, Almost Distributive Fuzzy Lattice, International Journal of Mathematics and its application, Vol. 5, Isue 1C (2017), pp. 307-316. 
[2] G. Epstein and A. Horn, P-algebras, an abstraction from post algebras, Algebra Universalis 4(1)(1974), pp. 195-206.

[3] Inheung Chon, Fuzzy partial order Relations and Fuzzy Lattices, Korean Journal of Mathematics 17 (2009), No. 4, pp. 361-374.

[4] I. Mezzemo, B. C. Bedregas, R. H. N. Santiago, Kinds of fuzzy lattices, second Brazilian congress on fuzzy system 2012, pp. 657-671.

[5] G. C. Rao, A. Berhanu, M. V. Ratnamani, Heyting Almost Distributive Lattices, International Journal of Computational Cognition 8(3) (2000), pp. 131-138.

[6] G. C. Rao, Naveen Kumar Kakuman, B-Almost Distributive Lattice, Southeast Asian Bulletin of Mathematics 39 (2015), pp. 545-554.

[7] G. C. Rao, Almost Distributive Lattice, Doctorial thesis, Department of Mathematics, Andra University, Visakhapatnam, 1980.

[8] U. M. Swammy and S. Ramesh, Birkhoff center of Almost Distributive Lattices, International Journal of Algebra 3 (2009), pp. 539-546.

[9] U. M. Swammy and G. C. Rao, Almost Distributive Lattices, Journal of the Australian Mathematical Society (Ser A) 31 (1981), pp. 77-91.

[10] U. M. Swamy, G. C. Rap and G. N. Rao, Pseudo-Complementation on Almost Distributive Lattices, Southeast Asian Bulletin of Mathematics 24 (2000), pp. 95-104.

Department of Mathematics

College of Science

Bahir Dar University

Bahir Dar, Ethiopia

e-mail: berhanu_assaye@yahoo.com

e-mail: mihretmahlet@yahoo.com

Department of Mathematics

College of Natural Science

Wollo University

Dessie, Ethiopia

e-mail: gerima233@gmail.com 